\title{
Rendezvous computed tomography-assisted endo- scopic needle-knife stricturotomy for sealed outlet of diverted large bowel
}

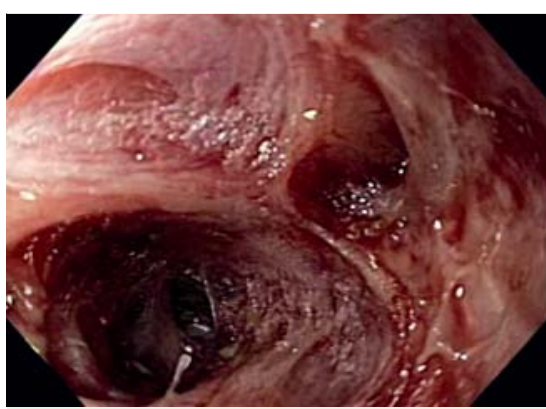

Fig. 1 Endoscopic view of completely sealed rectal stricture in a 27-year-old woman with a diverting end-ileostomy performed for severe Crohn's disease. Endoscopic balloon dilation and needle-knife stricturotomy were not possible and were risky for the patient.

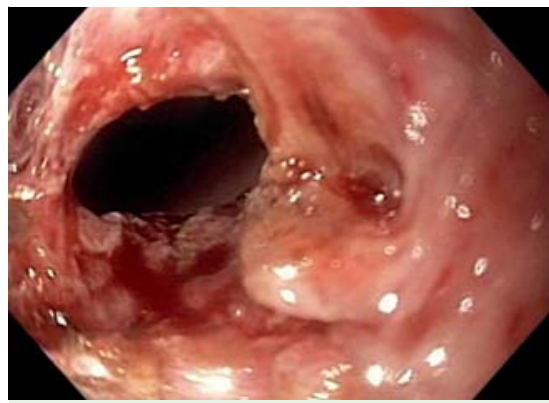

Fig. 3 Endoscopic view of successful stricturoplasty following CT-assisted needle knife stricturotomy in the distal diverted colon.

The patient was a 27-year-old woman, with a past medical history notable for 15 years of recalcitrant Crohn's disease with severe perianal fistulas and strictures, and anemia, psoriasis, and osteoporosis. Throughout, she had been treated with glucocorticoids for flare-ups and with antibiotics, surgical drainage, and seton placement for her perianal disease. Periodic remission was maintained with immunomodulators and antitumor necrosis factor (anti-TNF) biologic therapies throughout the disease course.

In 2009 she developed an ulcerated distal rectal stricture $1 \mathrm{~cm}$ in length, which was successfully treated with a wire-guided balloon (CRE; Boston Scientific, Marlborough, Massachusetts, USA). She underwent laparoscopic ileocolonic resection with a diverting end-ileostomy later in the same year for severe Crohn's disease and malnutrition, and because of failure

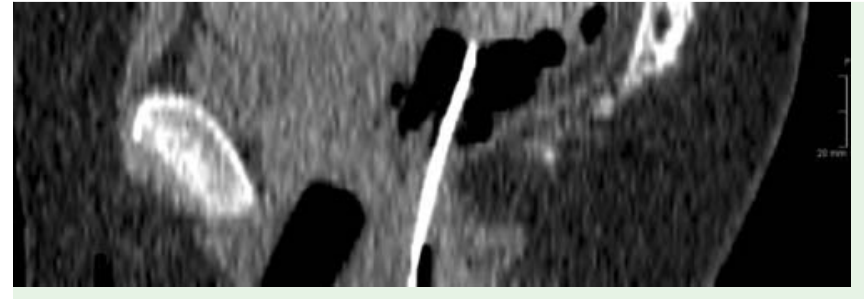

Fig. 2 Sagittal computed tomography (CT) image showing placement of a guidewire through the sealed rectal stricture into the patent portion of the distal rectal lumen.

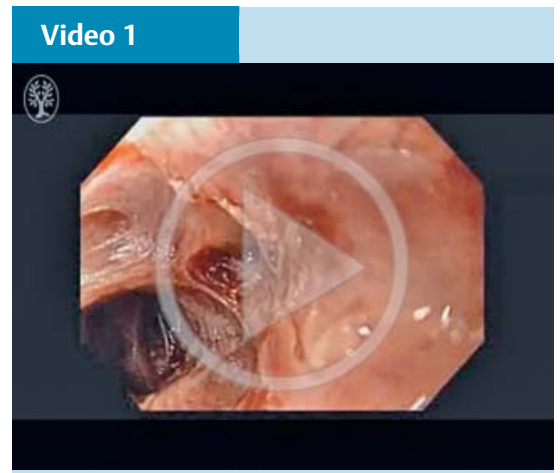

Failed attempts to pass a soft guidewire through a completely sealed rectal stricture in a 27-year-old woman with a diverting endileostomy performed for severe Crohn's disease. Endoscopic balloon dilation (EBD) or needle-knife stricturotomy were impossible.

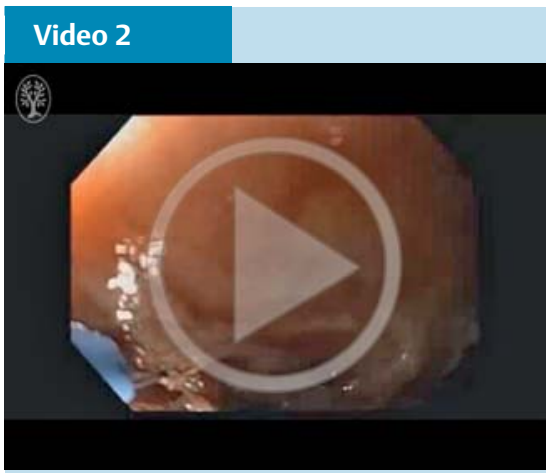

Part 1: Successful needle-knife stricturotomy of completely sealed rectal stricture with visualization of the lumen of the proximal diverted colon, following computed tomography (CT)guided placement of a guidewire. of treatment with adalimumab and certolizumab pegol. A significant portion of the large bowel remained in situ. Her luminal Crohn's disease went into remission postresection, but the perianal fistulas persisted, necessitating long-term therapy with ciprofloxacin and tinidazole in addition to placement of draining setons. Her perianal fistulas eventually healed with the medical therapy and fecal diversion.

She later developed psoriasis and psoriatic arthritis, while receiving adalimumab, and was switched to etanercept. Subsequent ileoscopy via the stoma showed a normal neodistal ileum. In July 2015 she presented with a completely sealed rectal stricture ( $\bullet$ Fig. 1). Attempts to pass a soft guidewire were futile and endoscopic balloon dilation (EBD) or needle-knife stricturotomy were impossible, as there was no access and the lumen of the diverted colon could not be visualized ( $\bullet$ Video 1 ).
It was then decided to create an access through computed tomography (CT)guided wire placement.

Using CT guidance, and with the patient under conscious sedation, an expert radiologist (J.C.V.) placed a 20-gauge Chiba needle through the stricture. Location of the needle tip in the distal rectum was confirmed by air insufflation. Then a 24-gauge modified Kopans wire (Cook Medical, Bloomington, Indiana, USA) was passed through the Chiba needle into the patent portion of the distal rectal lumen $(\bullet$ Fig. 2). The Chiba needle was withdrawn and the wire was secured to the skin externally. The patient was transported from the radiology suite to the outpatient endoscopy suite. With the patient under conscious sedation, the gastroenterology team (B.S., C.T.N.) performed endoscopic needle-knife stricturotomy along the course of the radio- 


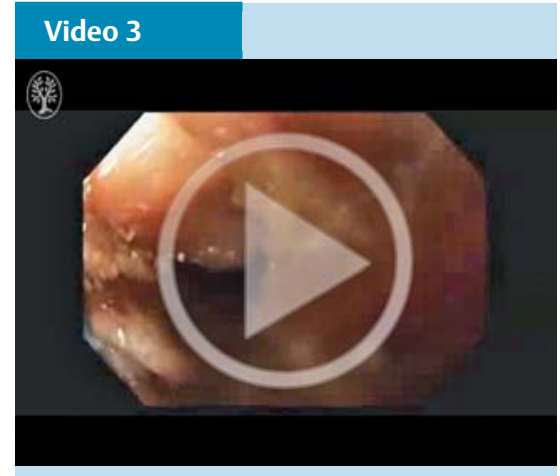

Part 2: Successful needle-knife stricturotomy of completely sealed rectal stricture with visualization of the lumen of the proximal diverted colon, following $\mathrm{CT}$-guided placement of a guidewire.

graphically placed metal guidewire (๑Video 2, $\bullet$ Video 3, $\bullet$ Video 4), making a patent access through the 2 -cm stricture ( $\bullet$ Fig.3). We passed a gastroscope through the previously strictured region without resistance. Mild diversion colitis with mucosal edema and multiple semiformed fecal bezoars were visualized. The large bezoars were removed endoscopi-

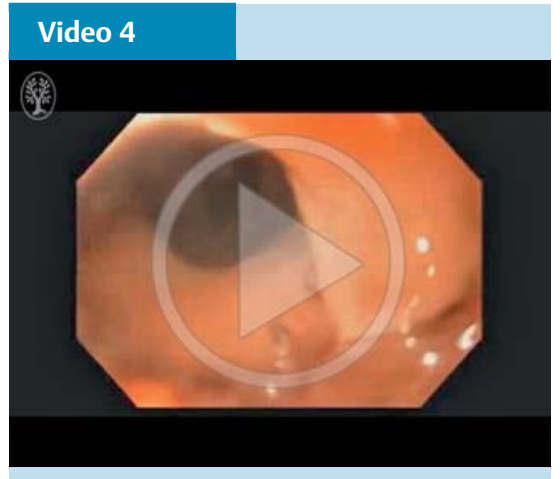

Part 3: Successful needle-knife stricturotomy of completely sealed rectal stricture with visualization of the lumen of the proximal diverted colon, following CT-guided placement of a guidewire.

cally. Normal saline was administered into the defunctionalized bowel, the patient reported being able to pass soft liquid substances within 24 hours, and the whole procedure was successful.

Endoscopy_UCTN_Code_TTT_1AS_2AZ

Competing interests: None

\section{Custon T. Nyabanga ${ }^{1}$, Joseph C. Veniero ${ }^{2}$, Bo Shen ${ }^{3}$}

${ }^{1}$ Cleveland Clinic Lerner College of Medicine, Cleveland, Ohio, USA

2 Department of Diagnostic Radiology, Cleveland Clinic, Cleveland, Ohio, USA

${ }^{3}$ Department of Gastroenterology/ Hepatology, Cleveland Clinic, Cleveland, Ohio, USA

Bibliography

DOI http://dx.doi.org/

10.1055/s-0034-1393676

Endoscopy 2015; 47: E625-E626

(c) Georg Thieme Verlag KG

Stuttgart · New York

ISSN 0013-726X

\section{Corresponding author \\ Bo Shen, MD}

Interventional IBD (i-IBD) Unit,

Center for Inflammatory Bowel Disease Digestive Disease Institute-A31, Cleveland Clinic 9500 Euclid Ave, Cleveland, OH 44195 USA

Fax: +1-216-444-6305

shenb@ccf.org 